\title{
Rough Set Approach for Group Evacuation Behavior Analysis in Passenger Transport Hub Area
}

\author{
Peng Chen, ${ }^{1}$ Xiaofeng $\mathrm{Ma},{ }^{2}$ and Shunying $\mathrm{Zhu}^{1}$ \\ ${ }^{1}$ School of Transportation, Wuhan University of Technology, Wuhan, Hubei 430063, China \\ ${ }^{2}$ Engineering Research Center for Transportation Safety (Ministry of Education), Wuhan University of Technology, \\ Wuhan, Hubei 430063, China \\ Correspondence should be addressed to Peng Chen; seupc@163.com
}

Received 3 November 2013; Revised 27 February 2014; Accepted 2 March 2014; Published 17 April 2014

Academic Editor: Huimin Niu

Copyright (C) 2014 Peng Chen et al. This is an open access article distributed under the Creative Commons Attribution License, which permits unrestricted use, distribution, and reproduction in any medium, provided the original work is properly cited.

\begin{abstract}
Evacuation behavior analysis is deemed to be one aspect of evacuation planning. However, existing studies have not discussed group evacuation decision-making in the face of disagreement among decision makers. In this paper, rough set theory is applied to analyze group evacuation decision-making in passenger transport hub area with various groups including kin, lover, friend, colleague, and classmate. In the approach, improved tabu search-based attribute reduction is proposed to find the minimal subset of attributes required to fully describe the information of group evacuation decision-making, and value reduction algorithm based on knowledge granulation is used to generate rules of group evacuation decision-making. Cross-validation procedure is adopted to estimate the performance of rough set theory. Experimental results indicate that rough set theory has favorable performance. Thus, the proposed approach provides a new way for evacuation behavior analysis.
\end{abstract}

\section{Introduction}

Urban passenger transport hub works as the joint of intermodal transit and the distribution center of massive passenger flow. In such densely populated area, small scale emergencies can result in severe consequences and should not be overlooked. It is vital to evacuate people from the affected area promptly. Thus, evacuation planning is very crucial. Evacuation behavior analysis is deemed to be one aspect of evacuation planning. Understanding emergency evacuation behavior would help better emergency evacuation planning.

Over the last few decades, considerable research has focused on evacuation behavior analysis related to hurricane evacuation [1-6] and building fire evacuation $[7,8]$. The majority of existing studies focused on individual and household evacuation decision-making and behavioral responses. However, it has not been discussed that how households arrive at a decision when decision-making of household members is inconsistent. As for emergency evacuation in passenger transport hub area, there are usually a large number of small groups with various relationships, including kin, lover, friend, colleague, and classmate. It is necessary to address the problem about group evacuation decisionmaking in the face of disagreement among decision makers.

By far, several methods have been put forward for helping us understand evacuation decision-making, including contingency table analysis [8], artificial neural network [7], and logistic regression analysis $[4,6,9,10]$. Among the above methods, contingency table analysis is only used to determine whether dependence exists between evacuation decision-making and related factors for a given significant level. Artificial neural network can establish the mapping function from explanatory variables to evacuation decisionmaking by using the multilayer perceptron, but it can only provide implied knowledge of evacuation decision-making. Logistic regression model is adopted to describe the relationship between explanatory variables and the probability of outcome of evacuation decision-making, but it needs to create the complex function. Thus rough set theory [11-13] 
TABLE 1: Chi-square test for group decision-making with several factors.

\begin{tabular}{lcccccccc}
\hline & Age & Gender & Education & Temperament & Experience & $\begin{array}{c}\text { Number of } \\
\text { luggage }\end{array}$ & $\begin{array}{c}\text { Familiar with } \\
\text { route }\end{array}$ & $\begin{array}{c}\text { Group } \\
\text { relationship }\end{array}$ \\
\hline Chi-square & 24.604 & 22.489 & 25.258 & 26.315 & 31.02 & 12.804 & 10.303 & 32.467 \\
$P$-value & 0.017 & 0.004 & 0.014 & 0.01 & 0.002 & 0.383 & 0.85 & 0.001 \\
\hline
\end{tabular}

is proposed to analyze group evacuation decision-making under emergency evacuation in passenger transport hub area. The approach adopted in this paper does not require the establishment of the function and can generate rules expressed in the form of if-then statements, which make knowledge have a clear meaning.

Rough set theory can remove redundant information through the reduction and extract decision rules from a large number of original data on the premise of the maintenance of the same classification ability. Knowledge reduction, including attribute reduction and value reduction, is one of the core issues in rough set theory. On the one hand, attribute reduction tends to reduce the complexity and cost of decision process and promote higher rule quality. In order to compute useful reduction of information systems, many researchers have developed some efficient algorithms based on computational intelligence tools of genetic algorithm [14], ant colony optimization [15], simulated annealing [15], particle swarm optimization [16], tabu search [17], and so on. On the other hand, value reduction is aimed at elimination of redundant attribute values in each rule and simplification of rules set while keeping the classification ability of rules set. Several algorithms have been proposed for value reduction, such as simplification rule algorithm [18], discernibility matrixbased algorithm [19], and heuristic algorithm based on mutual information [20]. In this paper, we propose improved tabu search-based attribute reduction (ITSAR) to find the minimal subset of attributes required to fully describe the information of group evacuation decision-making. Different from the existing research [17], we measure solution quality based on knowledge granulation because it can overcome the shortcomings of dependency degree [21] and use dynamic tabu tenure because it has better performance than fixed tabu tenure [22-24]. We also propose a heuristic algorithm based on knowledge granulation for value reduction, which is used to generate the rules of group evacuation decisionmaking.

The remainder of this paper is organized as follows. The next section describes evacuation behavior survey in Wuchang Railway Station area for the preparation of data set used in this study. In the following section, rough set theory is introduced, including related concepts, the algorithms for attribution reduction and value reduction, and evaluation of the approach. Section four presents the application of rough set theory on group evacuation decision-making and compares the proposed method with other methods in performance. Finally, we conclude the paper with a summary and outlook for further research.

\section{Evacuation Behavior Survey}

A survey was conducted about emergency evacuation behavior in Wuchang Railway Station area, with the hypothetical event of the toxic gas attack. The questionnaire was designed to collect the following information related to human behavior: (1) personal information including age, gender, education, temperament, and the number of luggage; (2) familiar with the route or not familiar with the route; (3) past experience; (4) the number of group members and group relationship; (5) human behavioral response including first action, evacuation route choice, group evacuation decisionmaking, and so on. Among the above information, the question for past experience is "Did you ever experience gas attack or participate in safety training," and structured answer is "(1) Never experience gas/training experience/knowledge, (2) Have gas experience/training experience/knowledge.” The structured answer for temperament is "(1) Choleric (You are a strong-willed individual who makes decisions quickly and decisively.), (2) Sanguine (You are affectionate, enjoy social activities, and make friends easily.), (3) Phlegmatic (You are dependable, polite, and even-tempered.), and (4) Melancholic (Time alone is vital for this reflective, introspective temperament.)".

A total of 952 interviews were performed and 909 valid replies were collected. There were 523 (57.5\%) valid replies coming from groups and 386 (42.5\%) from the single passenger. This paper focuses on the analysis of group evacuation decision-making in the face of disagreement among decision makers. In order to select the attributes influencing group evacuation decision-making, contingency table analysis was performed to test the correlation between group decisionmaking and the characteristics of individual and group by utilizing statistical analysis software SPSS 19.0. As shown in Table 1 , the results of $\chi^{2}$ (Chi-square) test at a significance value $(\alpha=0.05)$ indicate that there exists a significant relationship between group decision-making and several factors as follows: age, gender, education, temperament, experience, and group relationship. Based on group decision-making and the above factors, the attribute set and attribute value set are listed in Table 2.

\section{Rough Set Theory}

This section introduces rough set theory. Some basic notions are introduced in Section 3.1. Then the algorithms for attribute reduction and value reduction are developed in Sections 3.2 and 3.3, respectively. Section 3.4 explains evaluation of the approach. 
TABLE 2: The attribute set and attribute value set.

\begin{tabular}{|c|c|c|}
\hline Class & Attribute set & Attribute value set \\
\hline \multirow{6}{*}{ Condition attribute } & Age $(C 1)$ & (1) $<18$, (2) $18-35$, (3) $>35$ \\
\hline & Gender $(C 2)$ & (1) Female, (2) male \\
\hline & Education $(C 3)$ & (1) Senior high school or below (SHS), (2) junior college or above (JC) \\
\hline & Temperament $(C 4)$ & (1) Choleric, (2) sanguine, (3) phlegmatic, and (4) melancholic \\
\hline & Experience $(C 5)$ & $\begin{array}{l}\text { (1) Never experience gas/training experience/knowledge (NE), } \\
\text { (2) have gas experience/training experience/knowledge (HE) }\end{array}$ \\
\hline & Group relationship (C6) & (1) Kin, (2) lover, (3) friend, colleague, or classmate (FC) \\
\hline Decision attribute & Group decision-making $(D)$ & $\begin{array}{l}\text { (1) The minority is subordinate to the majority }(\mathrm{MSM}) \text {; (2) choose the } \\
\text { route approved by self (ABS); (3) choose the route approved by the } \\
\text { one familiar with the route }(\mathrm{ABF}) \text {; (4) choose the route approved by } \\
\text { the one doing things reasonably (ABR) }\end{array}$ \\
\hline
\end{tabular}

3.1. Preliminaries. In this section, some preliminary concepts such as indiscernibility, knowledge granulation, attribute reduction, and value reduction are briefly presented.

3.1.1. Indiscernibility. Let $S=(U, A)$ be an information system, where $U$, called universe, is a nonempty set of finite objects; $A$ is a nonempty finite set of attributes such that $a: U \rightarrow V_{a}$ for every $a \in A ; V_{a}$ is the value set of $a$. In a decision system with a set of decision attributes, $A=C \cup D$, where $C$ is the set of condition attributes and $D$ is the set of decision attributes. Such an information system also is called a decision table.

For an attribute set $P \subseteq A$, there is an associated indiscernibility relation $\operatorname{IND}(P)$ :

$$
\operatorname{IND}(P)=\left\{(x, y) \in U^{2} \mid \forall a \in P, a(x)=a(y)\right\} .
$$

$U / P$ denotes the partition of $U$ generated by $\operatorname{IND}(P)$. If $(x, y) \in \operatorname{IND}(P)$, then $x$ and $y$ are indiscernible by attributes from $P .[x]_{P}$ denotes the equivalence classes of the $P$-indiscernibility relation. The indiscernibility relation is the mathematical basis of rough set theory.

\subsubsection{Knowledge Granulation}

Definition 1. Let $S=(U, A)$ be an information system and $U / A=\left\{X_{1}, X_{2}, \ldots, X_{m}\right\}$; then knowledge granulation of $A$ is given by

$$
\mathrm{GK}(A)=\frac{1}{|U|^{2}} \sum_{i=1}^{m}\left|X_{i}\right|^{2},
$$

where the symbol $|\cdot|$ means the cardinality of a set.

Definition 2. Let $S=(U, C \cup D)$ be a decision table, where $U$ is the universe, $C$ is the set of conditional attributes and $D$ is the decision attribute, $B \subseteq C$; then the relative partition granularity of $B$ relative to $D$ is defined by Feng et al. [25]:

$$
\mathrm{GK}(D \mid B)=\mathrm{GK}(B)-\mathrm{GK}(B \cup D) .
$$

The value of $\operatorname{GK}(D \mid B)$ can be used to measure the classification ability of $B$ relative to $D$; that is, the larger the value of $\operatorname{GK}(D \mid B)$, the weaker the classification ability of $B$ relative to $D$.

Definition 3. Let $S=(U, C \cup D)$ be a decision table, where $U$ is the universe, $C$ is the set of conditional attributes, and $D$ is the decision attribute, $a \in C$; then the significance of attribute $a$ in $C$ relative to $D$ is defined by

$$
\operatorname{Sig}(a, C, D)=\mathrm{GK}(D \mid C-\{a\})-\mathrm{GK}(D \mid C) .
$$

3.1.3. Attribute Reduction. Attribute reduction in rough set theory can preserve the information content while reducing the number of attributes involved. Based on relative partition granularity, a relative reduct can be defined by the following definition.

Definition 4. Let $S=(U, C \cup D)$ be a decision table, where $U$ is the universe, $C$ is the set of conditional attributes, and $D$ is the decision attribute, $P \subseteq C$; if $\mathrm{GK}(D \mid P)=\mathrm{GK}(D \mid C)$ and $\operatorname{Sig}(a, P, D)>0, a \in P$, then $P$ is said to be a relative reduct of $C$ relative to $D$.

In particular, a relative reduct with minimal cardinality is called minimal reduct. The goal of attribute reduction is to find a minimal reduct.

3.1.4. Value Reduction. The process by which the maximum number of condition attribute values is removed without losing essential information is called value reduction. After value reduction, rules can be generated by associating the condition attribute values with the corresponding decision class value.

Definition 5. Let $S=(U, C \cup D)$ be a decision table, and let $X_{i} \in U\left|C, Y_{j} \in U\right| D$, and $X_{i} \cap Y_{j} \neq \varnothing$. By des $\left(X_{i}\right)$ and $\operatorname{des}\left(Y_{j}\right)$, we denote the descriptions of the equivalence classes $X_{i}$ and $Y_{j}$ in the decision table $S$. A decision rule is formally defined as

$$
Z_{i j}: \operatorname{des}\left(X_{i}\right) \longrightarrow \operatorname{des}\left(Y_{j}\right)
$$


Definition 6. The confidence of decision rule $Z_{i j}$ is defined as

$$
\operatorname{Con}\left(Z_{i j}\right)=\frac{\left|X_{i} \cap Y_{j}\right|}{\left|X_{i}\right|} .
$$

For a certain rule, $\operatorname{Con}\left(Z_{i j}\right)=1$, whereas an uncertain rule, $0<\operatorname{Con}\left(Z_{i j}\right)<1$.

3.2. Improved Tabu Search for Attribute Reduction. In this section, improved tabu search-based attribute reduction (ITSAR) is proposed to find a minimal reduct of group evacuation decision-making. First we introduce the main idea of tabu search, then describe the components of ITSAR, and finally give the ITSAR scheme.

3.2.1. Main Idea of Tabu Search. Tabu search (TS) is a metaheuristic optimization method originally proposed by Glover [26]. TS has been successfully applied in various fields $[23,24,27]$. The main ideas are to avoid recently visited parts of the solution space and to guide the search towards new and promising areas. Nonimproving moves are allowed to escape from local optima, and attributes of recently performed moves are declared tabu or forbidden for a number of iterations to avoid cycling. During the search, the algorithm maintains short-term and long-term memory structures. The short-term memory is built to keep the recency by constructing Tabu List (TL). The long-term memory is utilized to record solutions of special characters like elite and frequently visited solutions.

3.2.2. Solution Representation. ITSAR uses a binary representation for solutions (attribute subsets). Therefore, a trial solution $x$ is a $0-1$ vector with dimension equal to the number of condition attributes $|C|$. If a component $x_{i}$ of $x, i=$ $1, \ldots,|C|$, has the value 1 , then the $i$ th attribute is contained in the attribute subset represented by the trial solution $x$. Otherwise, the solution $x$ does not contain the $i$ th attribute.

3.2.3. Solution Quality Measure. $\operatorname{GK}(D \mid x)$ means the relative partition granularity of solution $x$ relative to decision attribute $D$. Comparing two solutions $x$ and $x^{\prime}$, we say $x$ is better than $x^{\prime}$ if one of the following conditions holds:

$$
\begin{gathered}
\mathrm{GK}(D \mid x)<\mathrm{GK}\left(D \mid x^{\prime}\right), \\
\sum_{i} x_{i}<\sum_{i} x_{i}^{\prime} \quad \text { if GK }(D \mid x)=\mathrm{GK}\left(D \mid x^{\prime}\right) .
\end{gathered}
$$

3.2.4. Tabu List. The role of Tabu List (TL) is to avoid being trapped in local optima. The first and second positions in TL are permanently reserved for two special solutions: solution of all ones (i.e., all attributes are considered), and solution of all zeroes (i.e., all attributes are discarded). The remaining positions in TL are used to save the most recently visited solutions. To improve search performance, dynamic selection strategies of tabu tenure are as follows.

The range of tabu tenure $t$ is defined by parameters $t_{\min }$ and $t_{\max }$. The initial tabu tenure is set equal to round $\left(\left(t_{\min }+t_{\max }\right) / 2\right)$. In the process of the implementation of diversification strategy, the tabu tenure is randomly selected within the range $\left[t_{0}+1, t_{\max }\right]$, following a uniform distribution. In the course of the implementation of intensification strategy, the tabu tenure is randomly selected within the range $\left[t_{\min }, t_{0}\right]$, following a uniform distribution. If there are no improvements in $0.75 I_{\text {imp }}$ iterations ( $I_{\text {imp }}$ means max number of consecutive nonimprovement iterations), the tabu tenure is randomly selected within the range $\left[t_{\min }, t_{\max }\right]$, following a uniform distribution.

3.2.5. Neighborhood Trials Generation. Trial solutions $y^{j}, j=$ $1, \ldots, l$, are generated by changing $j$ positions in current solution $x$ randomly based on tabu restrictions as in the following procedure.

Procedure $1\left(\left[y^{1}, \ldots, y^{l}\right]=\operatorname{Trials}(x, \mathrm{TL}, l)\right)$.

(1) Repeat the following steps for $j=1, \ldots, l$.

(2) Set $y^{j}=x$, and choose $j$ random positions $p_{1}, \ldots, p_{j}$ of $y^{j}$.

(3) Update the chosen positions by the rule $y_{P_{i}}^{j}=1-y_{P_{i}}^{j}$, $i=1, \ldots, j$.

(4) If $y^{j} \in T L$, then return to Step 2 to generate another $y^{j}$.

3.2.6. Diversification Strategy. The main roles of diversification strategy are to direct the search process to new solution regions and to accelerate escaping from local optima. ITSAR defines a vector $v^{F}$ of dimension $|C|$ which counts the numbers of choosing each condition attribute among the generated trial solutions. Then, a diverse solution $x^{\mathrm{div}}$ can be generated to contain attributes chosen with probability inversely proportional to their appearance in $v^{F}$. The procedure is as follows.

Procedure $2\left(\left[x^{\mathrm{div}}\right]=\operatorname{Diverse}\left(v^{F}\right)\right)$.

(1) Generate random numbers $r_{1}, \ldots, r_{|C|} \in(0,1)$.

(2) Repeat the following step for $i=1, \ldots,|C|$.

(3) If $r_{i}>v_{i}^{F} / \sum_{i=1}^{|C|} v_{i}^{F}$, set $x^{\mathrm{div}}=1$. Otherwise, set $x^{\mathrm{div}}=$ 0 .

3.2.7. Intensification Strategy. If the search still cannot find any improvement during some iterations after generating $x^{\text {div }}$, ITSAR applies an intensification strategy to refine the best reduct $x^{\text {best }}$ found so far. The best reduct $x^{\text {best }}$ refinement, called Shaking, tries to reduce the attributes contained in $x^{\text {best }}$ one by one without increasing $\operatorname{GK}\left(D \mid x^{\text {best }}\right)$. The search is continued from $x^{\text {best }}$ no matter whether it can be improved through the Shaking Mechanism or not. Finally, the search process is terminated and a final refinement is applied. The procedure is as follows. 
Procedure 3 (Shaking $\left(x^{\text {best }}\right)$ ).

(1) Construct the set $W$ of all positions of ones in $x^{\text {best }}$; that is, the elements of $W$ represent the attributes contained in $x^{\text {best }}$.

(2) Repeat the following steps for $j=1, \ldots,|W|$.

(3) Delete the attribute $w_{j} \in W$, and compute a relative partition granularity.

(4) Update $x^{\text {best }}\left(x_{w_{j}}^{\text {best }}=0\right)$ if relative partition granularity is decreased or if relative partition granularity remains the same but the number of the attributes contained in reducts is decreased.

3.2.8. ITSAR Algorithm. The complete algorithm is as follows.

(1) Let the Tabu List (TL) contain the two extreme solutions: solution of all ones and solution of all zeroes; set $v^{F}$ to be a zero vector. Choose an initial solution $x_{0}$, and set the counter $k=0$. Select $I_{\max }$, $I_{\text {imp }}, I_{\text {shak }}$, and $I_{\text {div }}$ such that $I_{\max }>I_{\text {imp }}>I_{\text {shak }}>I_{\text {div }}$.

(2) Generate neighborhood trials $y^{1}, \ldots, y^{l}$ around $x^{k}$ using Procedure 1.

(3) Set $x^{k+1}$ equal to the best trial solution from $y^{1}, \ldots, y^{l}$, and update TL, $v^{F}$, and $x^{\text {best }}$. Set $k=k+1$.

(4) If the number of iterations exceeds $I_{\max }$ or the number of iterations without improvement exceeds $I_{\mathrm{imp}}$, terminate the search.

(5) If the number of iterations without improvement exceeds $I_{\text {shak }}$, apply Procedure 3 to improve $x^{\text {best }}$, set $x^{k}=x^{\text {best }}$, and go to Step 2.

(6) If the number of iterations without improvement exceeds $I_{\text {div }}$, apply Procedure 2 to obtain a new diverse solution $x^{\text {div }}$, set $x^{k}=x^{\text {div }}$, and go to Step 2 .

3.3. Value Reduction Based on Knowledge Granulation. A heuristic algorithm based on knowledge granulation for value reduction, which is used to generate decision rules of group evacuation decision-making, is described as follows.

(1) Examine the condition attribute of each decision rule by the column; if removing a condition attribute, three possible cases are as follows:

(1) if there are conflicting decision rules, then retain the dropped attribute value of conflicting decision rules, which means the value cannot be eliminated;

(2) if there are duplicate decision rules, then mark the dropped attribute value of duplicate decision rules as "*", which means the value can be eliminated;

(3) if there are no conflicting and duplicate decision rules, then mark the dropped attribute value as "?", which means whether the value can be eliminated is pending.
(2) Delete possible duplicate decision rules. If all the condition attributes of a decision rule are marked, then change the attribute value marked with "?" to the original attribute value.

(3) Examine the attribute value marked with "?" of each decision rule.

(1) If there is only one "?", go to (3); if there are more than one "?", calculate the significance of all attribute values marked with "?" according to Definition 3.

(2) Select the attribute value marked with "?" and maximum of the significance in the decision rule

(3) If the decision can be made only by the attribute value without the mark, go to (4); otherwise, go to (5).

(4) Change the attribute value marked with "?" to “*”.

(5) Change the attribute value marked with "?" to the original attribute value, and go to (2).

(4) Delete decision rules in which all the condition attributes are marked as "*” and the possible duplicate decision rules.

(5) If there are two decision rules which satisfy the following two conditions: (a) only one condition attribute value is different, (b) one of different attribute values is marked as "*", then, for the decision rule in which different attribute values is marked as “*”, if the decision can be made by the attribute values without the mark, delete another decision rule; otherwise, delete this rule.

(6) Calculate the confidence of each rule; export the rules.

3.4. Evaluation of the Approach. In this study, examples were scarce; thus, cross-validation (CV) procedure [28] was used to evaluate the performance of the approach. A $k$-fold cross-validation procedure partitions the data into $k$ disjoint subsets of nearly equal size. One of the subsets is reserved for testing, whereas the rest of the data constitute the training sample. This procedure is repeated $k$ times. Each time using a different subset as the test set, and the final result is the arithmetic average of $k$ separate tests.

We evaluated the performance of the approach by applying 10 times 5-fold cross-validation tests. The performance of the approach was measured by the hit rate of decision rule with maximum value of confidence $\left(\right.$ hit $\left._{0}\right)$, the hit rate of decision rule with maximum value of confidence and second largest value of confidence ( hit $_{1}$ ), and the comprehensive hit rate $\left(\mathrm{hit}_{2}\right)$ computed by

$$
\begin{gathered}
\text { hit }_{0}=\frac{c_{0}}{t}, \quad \text { hit }_{1}=\frac{c_{0}+c_{1}}{t}, \\
\text { hit }_{2}=0.6 \text { hit }_{0}+0.4 \text { hit }_{1},
\end{gathered}
$$

where $c_{0}$ is the number of instances in the test set which can be correctly classified by decision rule with maximum value of 
TABle 3: An example of decision table with eight objects.

\begin{tabular}{|c|c|c|c|c|c|c|c|}
\hline Objects & $C 1$ & $C 2$ & C3 & $C 4$ & $C 5$ & C6 & $D$ \\
\hline 1 & 2 & 1 & 2 & 2 & 1 & 2 & 2 \\
\hline 2 & 2 & 1 & 1 & 3 & 1 & 1 & 3 \\
\hline 3 & 2 & 2 & 2 & 2 & 2 & 1 & 4 \\
\hline 4 & 2 & 1 & 1 & 2 & 1 & 3 & 1 \\
\hline 5 & 3 & 2 & 1 & 3 & 1 & 3 & 1 \\
\hline 6 & 1 & 2 & 1 & 2 & 2 & 1 & 2 \\
\hline 7 & 2 & 2 & 2 & 4 & 2 & 3 & 1 \\
\hline 8 & 1 & 2 & 1 & 2 & 2 & 3 & 3 \\
\hline
\end{tabular}

confidence, $c_{1}$ is the number of instances in the test set which can be correctly classified by decision rule with second largest value of confidence, $t$ is the total number of instances in the test set. The comprehensive hit rate $\left(\mathrm{hit}_{2}\right)$ was also used as the model selection criterion.

\section{Application to Group Evacuation Decision-Making}

This section presents our tests on group evacuation decisionmaking. We firstly develop the decision table in Section 4.1. Then we extract the reducts with the application of attribute reduction in Section 4.2. Based on the reducts, a set of decision rules are generated by means of value reduction in Section 4.3. Section 4.4 presents the testing results of the rules. Lastly, we compare the proposed method with other methods in performance.

4.1. Representation of Decision Table. The first step is to develop decision table for group evacuation decision-making. As discussed previously, we have used the dataset from evacuation behavior survey in Wuchang Railway Station area. The decision table includes 523 objects or samples. For each record, six conditional attributes are registered.

Table 3 shows eight objects of group evacuation decisionmaking used as the example of decision table.

4.2. Reduction of Attributes. The algorithms for attribute reduction and value reduction were programmed in MATLAB and applied to the decision table of group evacuation decision-making. The parameter values used in ITSAR were set to the following values: $t=[5,10], l=3, I_{\max }=100$, $I_{\text {imp }}=40, I_{\text {shak }}=20, I_{\text {div }}=10$. These chosen values are based on the common setting in $[17,29]$. This indicates the potential for future improvement of ITSAR by systematically finetuning these parameters using statistical tests as suggested by $\mathrm{Xu}$ et al. [30].

After attribute reduction by applying 10 times 5-fold cross-validation tests, some reducts can be obtained. Table 4 shows the frequency of individual reduct occurring among the set of reducts. Evidently, there is one common attribute, that is, group relationship (C6), occurring in all the reducts.
TABLE 4: Reducts and their frequency.

\begin{tabular}{lc}
\hline Reducts & Frequency \\
\hline$\{C 1, C 4, C 6\}$ & 8 \\
$\{C 2, C 3, C 6\}$ & 2 \\
$\{C 2, C 4, C 6\}$ & 14 \\
$\{C 4, C 6\}$ & 11 \\
$\{C 2, C 3, C 4, C 6\}$ & 6 \\
$\{C 1, C 2, C 4, C 6\}$ & 5 \\
$\{C 3, C 4, C 6\}$ & 3 \\
$\{C 1, C 6\}$ & 1 \\
\hline
\end{tabular}

4.3. Decision Rules. Based on reducts obtained in the previous step, decision rules can be generated from the decision table by value reduction. For the reduct with the highest frequency, rules are obtained from the corresponding training set and shown in Table 5. In these decision rules, 2 rules are certain and others are uncertain. For example, rule 1 and rule 3 are selected to describe below.

Rule 1 means that if condition attribute values satisfy the following conditions, that is, gender is male and temperament is choleric, and group relationship is lover, then group decision-making mode chosen by individual is choosing the route approved by the one doing things reasonably. The confidence of this rule is 1 .

Rule 3 means that if condition attribute values satisfy the following conditions, that is, gender is female and temperament is choleric, and group relationship is lover, then group decision-making mode chosen by individual has three possibilities, that is, choosing the route approved by self (with the confidence of 0.125 ), or choosing the route approved by the one familiar with the route (with the confidence of 0.5 ), or choosing the route approved by the one doing things reasonably (with the confidence of 0.375 ).

4.4. Results of Testing. Decision rules generated from the training set are applied to the corresponding testing set in order to harvest a performance estimate. The results from 10 times 5-fold cross-validation tests show that the range

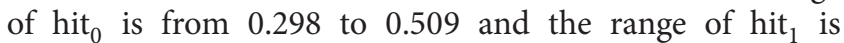
from 0.529 to 0.759 . As shown in Figure 1, the majority of hit $_{0}$ is above 0.4 and the majority of hit ${ }_{1}$ is above 0.6. For 
TABLE 5: Decision rules of the corresponding sample.

\begin{tabular}{lc}
\hline Decision rules & Confidence \\
\hline Rule 1: If $(C 2=2)$ and $(C 4=1)$ and $(C 6=2)$, then $(D=4)$ & 1 \\
Rule 2: If $(C 2=2)$ and $(C 4=4)$ and $(C 6=2)$, then $(D=3)$ & 1 \\
Rule 3: If $(C 2=1)$ and $(C 4=1)$ and $(C 6=2)$, then $(D=2$ or 3 or 4$)$ & $(0.125,0.5,0.375)$ \\
Rule 4: If $(C 4=2)$, then $(D=1$ or 2 or 3 or 4$)$ & $(0.241,0.177,0.418,0.164)$ \\
Rule 5: If $(C 2=1)$ and $(C 6=1)$, then $(D=1$ or 2 or 3 or 4$)$ & $(0.286,0.428,0.286)$ \\
Rule 6: If $(C 2=1)$ and $(C 4=3)$ and $(C 6=2)$, then $(D=1$ or 2 or 4$)$ & $(0.333,0.667)$ \\
Rule 7: If $(C 2=1)$ and $(C 4=4)$ and $(C 6=2)$, then $(D=2$ or 4$)$ & $(0.25,0.5,0.25)$ \\
Rule 8: If $(C 2=1)$ and $(C 4=4)$ and $(C 6=3)$, then $(D=1$ or 2 or 4$)$ & $(0.13,0.208,0.532,0.13)$ \\
Rule 9: If $(C 2=2)$ and $(C 4=3)$, then $(D=1$ or 2 or 3 or 4$)$ & $(0.143,0.571,0.286)$ \\
Rule 10: If $(C 2=2)$ and $(C 4=4)$ and $(C 6=1)$, then $(D=1$ or 3 or 4$)$ & $(0.167,0.167,0.666)$ \\
Rule 11: If $(C 2=2)$ and $(C 4=4)$ and $(C 6=3)$, then $(D=1$ or 2 or 4$)$ & $(0.148,0.185,0.519,0.148)$ \\
Rule 12: If $(C 4=1)$ and $(C 6=1)$, then $(D=1$ or 2 or 3 or 4$)$ & $(0.207,0.276,0.31,0.207)$ \\
Rule 13: If $(C 4=1)$ and $(C 6=3)$, then $(D=1$ or 2 or 3 or 4$)$ & $(0.175,0.222,0.539,0.064)$ \\
Rule 14: If $(C 4=3)$ and $(C 6=3)$, then $(D=1$ or 2 or 3 or 4$)$ &
\end{tabular}

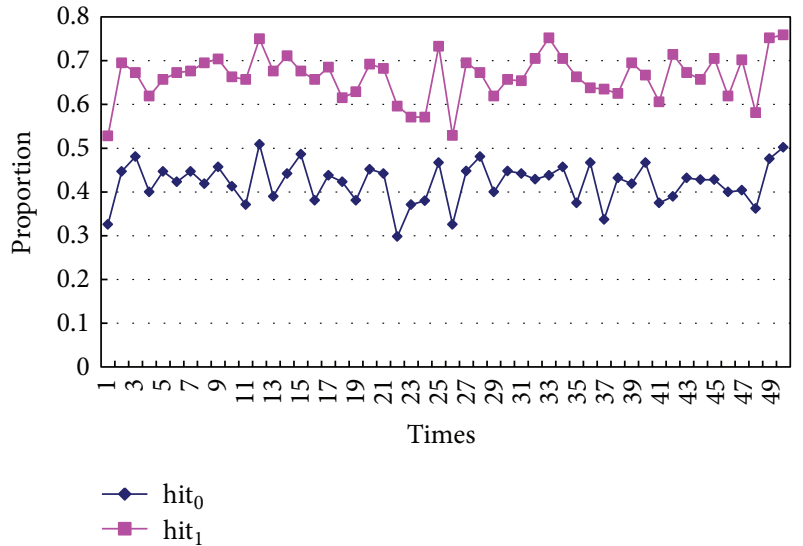

Figure 1: Performance of rough set theory.

the best rough set model, hit $_{0}$, hit , and hit $_{2}$ are 0.502, 0.759, and 0.605 , respectively. The best rough set model consists of three conditional attributes: gender, temperament, and group relationship.

4.5. Comparison with Other Methods. To get a better picture of the power of rough set theory, a comparison with other techniques using the same training and testing samples would prove useful. For the purpose of comparison, we chose tabu search for attribute reduction (TSAR) [17] and multinomial logistics regression (MLR) [31].

In the TSAR algorithm, the dependency degree of decision attribute is used to measure the quality of a solution, and fixed tabu tenure is used. We set fixed tabu tenure as 8 in our study. TSAR and ITSAR could obtain the same reducts in this paper. The solution times of two methods for 50 runs are displayed in Figure 2. Regarding average solution time, ITSAR obtained the reducts in $24.7 \%$ less time than TSAR. The average solution times were 0.827 CPU seconds for ITSAR and 1.1 CPU seconds for TSAR.

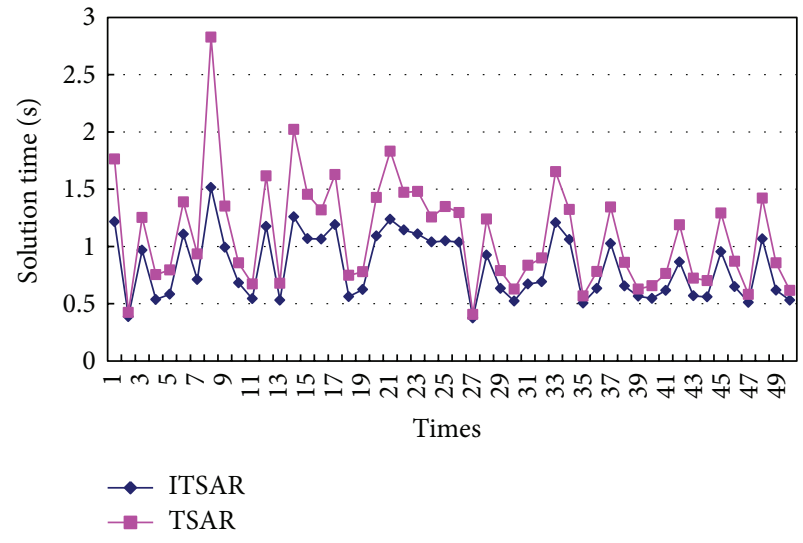

FIGURE 2: Solution times of ITSAR and TSAR.

Multinomial logistics regression can be used when a categorical dependent variable has more than two categories. For the implementation of the multinomial logistic regression model, the backward elimination procedure was performed by using SPSS software in this study. The performance of multinomial logistics regression model was determined by cross-validation procedure described in Section 3.4. Here, in (8), $c_{0}$ is the number of instances in the test set which can be correctly classified by the category with maximum value of probability, and $c_{1}$ is the number of instances in the test set which can be correctly classified by the category with the second largest value of probability. The best logistic regression model was extracted on the basis of goodness-of-fit test and the hit rates.

The results from 10 times 5-fold cross-validation test show that the range of $\mathrm{hit}_{0}$ is from 0.123 to 0.48 and the range of hit $_{1}$ is from 0.451 to 0.723 . As shown in Figure 3, the majority of hit ${ }_{0}$ is below 0.4 and the majority of hit $_{1}$ is above 0.6 . For the best logistic regression model, hit $_{0}$, hit ${ }_{1}$, and hit 2 are 0.48 , 0.693 , and 0.565 , respectively. Goodness-of-fit measures of the best logistic regression model are displayed in Table 6. 




FIgURE 3: Performance of multinomial logistics regression.

TABLE 6: Goodness-of-fit measures of the best logistic regression model.

\begin{tabular}{lccc}
\hline & $\chi^{2}$ & DF & Sig. \\
\hline Pearson & 401.191 & 360 & 0.066 \\
Deviance & 385.701 & 360 & 0.168 \\
\hline
\end{tabular}

TABLE 7: Performance comparison.

\begin{tabular}{lcc}
\hline & hit $_{0}$ & hit $_{1}$ \\
\hline Rough set theory & 0.421 & 0.663 \\
MLR & 0.362 & 0.651 \\
\hline
\end{tabular}

The best logistic regression model consists of three variables: gender, temperament, and group relationship, which is the same as conditional attributes in the best rough set model.

Table 7 depicts the average results of 10 times 5-fold cross-validation test for two approaches. The results of the approach comparison show that rough set theory method is superior to multinomial logistics regression in terms of testing performance.

On the other hand, the fluctuation of the curve in Figure 1 is relatively moderate, whereas the fluctuation of the curve in Figure 3 is relatively marked. The comparisons suggest that the stability of testing performance for rough set theory method is better than that for multinomial logistics regression.

\section{Conclusions}

In this paper, we focus on the analysis of group evacuation decision-making in the face of disagreement among decision makers in passenger transport hub area. Rough set theory is applied to analyze group evacuation decisionmaking. Based on evacuation behavior survey, we develop the decision table of group evacuation decision-making. An improved tabu search-based attribute reduction (ITSAR) is proposed to find a minimal reduct of decision table, and then a heuristic algorithm based on knowledge granulation for value reduction is introduced for rule extraction of decision table. According to the presented research, rules of group evacuation decision-making are generated in a readily understandable form (a set of simple if-then statements). By using 10 times 5-fold cross-validation tests, we compare the proposed method with other methods in performance. The results show that ITSAR outperformed TSAR in terms of solution time, and rough set theory has the advantage over multinomial logistics regression for the analysis of group evacuation decision-making. It can be concluded that rough set theory can quickly obtain more simple decision rules of group evacuation decision-making and provide a new way for evacuation behavior analysis.

Further research mainly includes two aspects. First, it is worthwhile to develop effective update method for rule database after increasing new samples. Second, this model could be integrated with a larger set of behavioral models into an agent-based simulation framework to comprehensively model the evacuation process, which would help public agencies develop evacuation plans that align with evacuee choices and behavior.

\section{Conflict of Interests}

The authors declare that there is no conflict of interests regarding the publication of this paper.

\section{Acknowledgments}

Research for this study was supported by the National Natural Science Foundation of China (51208400, 51108362, and 51078299) and the Fundamental Research Funds for the Central Universities of China (no. 2011-IV-125).

\section{References}

[1] M. K. Lindell, J.-C. Lu, and C. S. Prater, "Household decision making and evacuation in response to Hurricane Lili," Natural Hazards Review, vol. 6, no. 4, pp. 171-179, 2005.

[2] N. Dash and H. Gladwin, "Evacuation decision making and behavioral responses: individual and household," Natural Hazards Review, vol. 8, no. 3, pp. 69-77, 2007.

[3] J. Czajkowski, "Is it time to go yet? Understanding household hurricane evacuation decisions from a dynamic perspective," Natural Hazards Review, vol. 12, no. 2, pp. 72-84, 2011.

[4] S. Hasan, S. Ukkusuri, H. Gladwin, and P. Murray-Tuite, "Behavioral model to understand household-level hurricane evacuation decision making," Journal of Transportation Engineering, vol. 137, no. 5, pp. 341-348, 2011.

[5] M. K. Lindell, J. E. Kang, and C. S. Prater, "The logistics of household hurricane evacuation," Natural Hazards, vol. 58, no. 3, pp. 1093-1109, 2011.

[6] R. Mesa-Arango, S. Hasan, S. V. Ukkusuri, and P. Murray-Tuite, "Household-Level model for hurricane evacuation destination type choice using hurricane ivan data," Natural Hazards Review, vol. 14, no. 1, pp. 11-20, 2013.

[7] S. M. Lo, M. Liu, P. H. Zhang, and R. K. K. Yuen, "An artificial neural-network based predictive model for pre-evacuation human response in domestic building fire," Fire Technology, vol. 45, no. 4, pp. 431-449, 2009. 
[8] C. M. Zhao, S. M. Lo, S. P. Zhang, and M. Liu, "A post-fire survey on the pre-evacuation human behavior," Fire Technology, vol. 45, no. 1, pp. 71-95, 2009.

[9] H. Fu and C. G. Wilmot, "Sequential logit dynamic travel demand model for hurricane evacuation," Transportation Research Record, no. 1882, pp. 19-26, 2004.

[10] Y. Hsu and S. Peeta, "An aggregate approach to model evacuee behavior for no-notice evacuation operations," Transportation, vol. 40, no. 3, pp. 671-696, 2013.

[11] Z. Pawlak, "Rough sets," International Journal of Computer and Information Sciences, vol. 11, no. 5, pp. 341-356, 1982.

[12] P. Sawicki and J. Zak, "Technical diagnostic of a fleet of vehicles using rough set theory," European Journal of Operational Research, vol. 193, no. 3, pp. 891-903, 2009.

[13] J.-Y. Shyng, H.-M. Shieh, G.-H. Tzeng, and S.-H. Hsieh, “Using FSBT technique with Rough Set Theory for personal investment portfolio analysis," European Journal of Operational Research, vol. 201, no. 2, pp. 601-607, 2010.

[14] L.-Y. Zhai, L.-P. Khoo, and S.-C. Fok, "Feature extraction using rough set theory and genetic algorithms - an application for the simplification of product quality evaluation," Computers and Industrial Engineering, vol. 43, no. 4, pp. 661-676, 2002.

[15] R. Jensen and Q. Shen, "Semantics-preserving dimensionality reduction: rough and fuzzy-rough-based approaches," IEEE Transactions on Knowledge and Data Engineering, vol. 16, no. 12, pp. 1457-1471, 2004.

[16] X. Wang, J. Yang, X. Teng, W. Xia, and R. Jensen, "Feature selection based on rough sets and particle swarm optimization," Pattern Recognition Letters, vol. 28, no. 4, pp. 459-471, 2007.

[17] A.-R. Hedar, J. Wang, and M. Fukushima, "Tabu search for attribute reduction in rough set theory," Soft Computing, vol. 12, no. 9, pp. 909-918, 2008.

[18] A.-E. Hassanien, "Rough set approach for attribute reduction and rule generation: a case of patients with suspected breast cancer," Journal of the American Society for Information Science and Technology, vol. 55, no. 11, pp. 954-962, 2004.

[19] X. Bai, M. Zhang, Y. Qiu, and Q. Wu, "Algorithm for decision rules reduction in incomplete information system based on binary discemibility matrix," in Proceedings of the IEEE International Conference on Mechatronics and Automation (ICMA '09), pp. 4061-4066, August 2009.

[20] L. Zhang, X. Lu, H. Wu, and S. Hao, "Improved heuristic algorithm used in attribute value reduction of rough set," Chinese Journal of Scientific Instrument, vol. 30, no. 1, pp. 8285, 2009.

[21] J. Liang, J. Wang, and Y. Qian, "A new measure of uncertainty based on knowledge granulation for rough sets," Information Sciences, vol. 179, no. 4, pp. 458-470, 2009.

[22] E. Rolland, D. A. Schilling, and J. R. Current, "An efficient tabu search procedure for the p-Median Problem," European Journal of Operational Research, vol. 96, no. 2, pp. 329-342, 1997.

[23] P. Yin and Y. Guo, "Optimization of multi-criteria website structure based on enhanced tabu search and web usage mining," Applied Mathematics and Computation, vol. 219, no. 24, pp. 11082-11095, 2013.

[24] Q. Wu and J.-K. Hao, "An adaptive multistart tabu search approach to solve the maximum clique problem," Journal of Combinatorial Optimization, vol. 26, no. 1, pp. 86-108, 2013.

[25] Q. Feng, D. Miao, and Y. Cheng, "An approach to knowledge reduction based on relative partition granularity," in Proceedings of the IEEE International Conference on Granular Computing (GRC '08), pp. 226-231, August 2008.
[26] F. Glover, "Future paths for integer programming and links to artificial intelligence," Computers \& Operations Research, vol. 13, no. 5, pp. 533-549, 1986.

[27] C. Xie and M. A. Turnquist, "Lane-based evacuation network optimization: an integrated Lagrangian relaxation and tabu search approach," Transportation Research C: Emerging Technologies, vol. 19, no. 1, pp. 40-63, 2011.

[28] P. A. Berger, "Rough set rule induction for suitability assessment," Environmental Management, vol. 34, no. 4, pp. 546-558, 2004.

[29] C. K. Y. Lin and R. C. W. Kwok, "Multi-objective metaheuristics for a location-routing problem with multiple use of vehicles on real data and simulated data," European Journal of Operational Research, vol. 175, no. 3, pp. 1833-1849, 2006.

[30] J. Xu, S. Y. Chiu, and F. Glover, "Fine-tuning a tabu search algorithm with statistical tests," International Transactions in Operational Research, vol. 5, no. 3, pp. 233-244, 1998.

[31] G. H. Bham, B. S. Javvadi, and U. R. R. Manepalli, "Multinomial logistic regression model for Single-Vehicle and multivehicle collisions on urban u.s. Highways in arkansas," Journal of Transportation Engineering-Asce, vol. 138, no. 6, pp. 786-797, 2012. 




Advances in

Operations Research

mansans



The Scientific World Journal
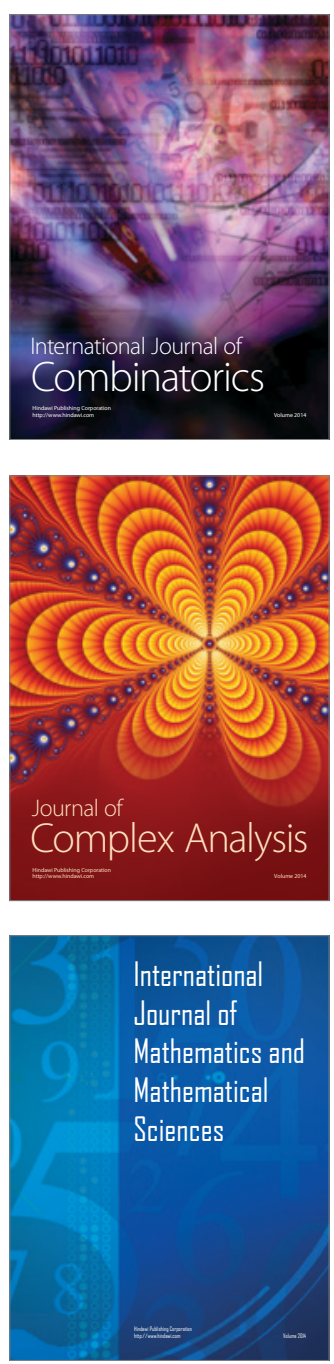
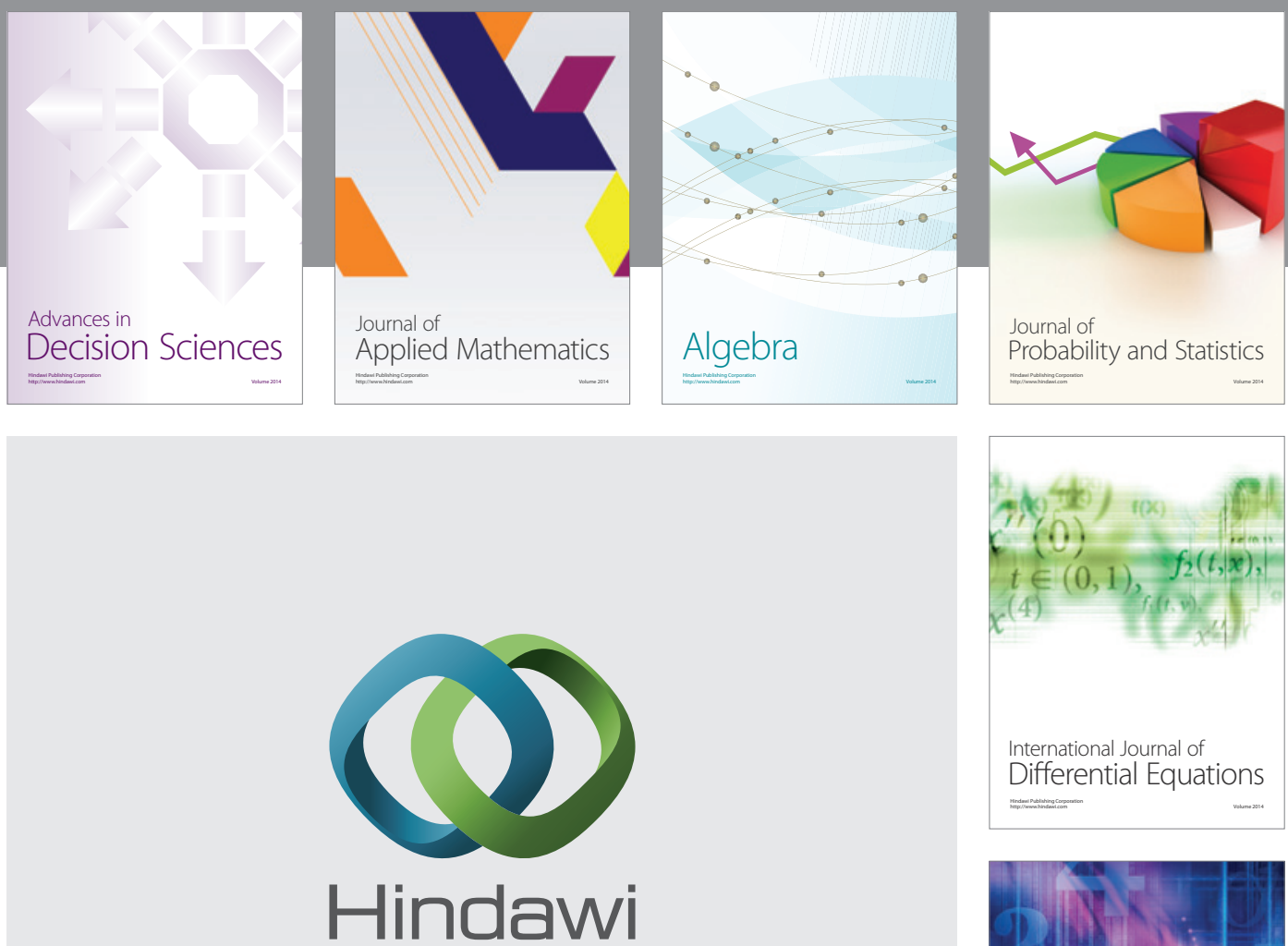

Submit your manuscripts at http://www.hindawi.com
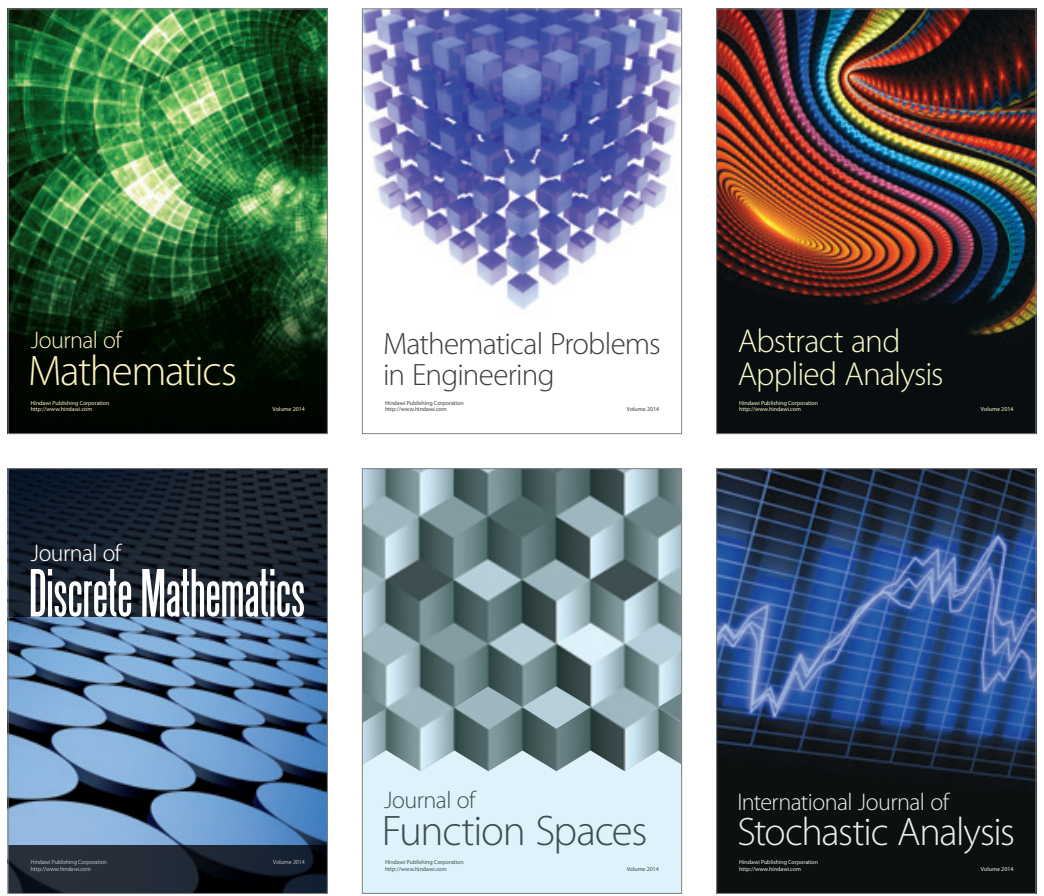

Journal of

Function Spaces

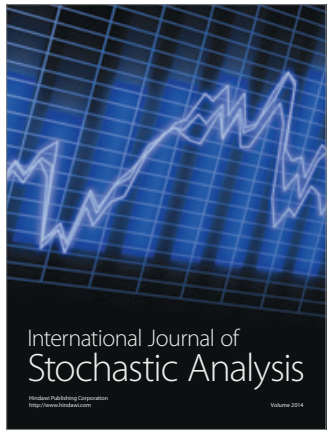

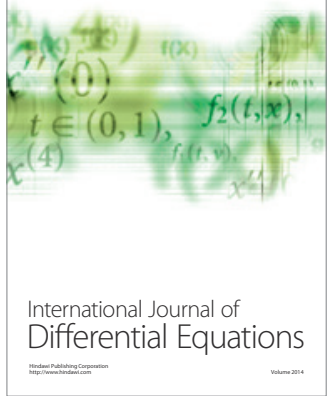
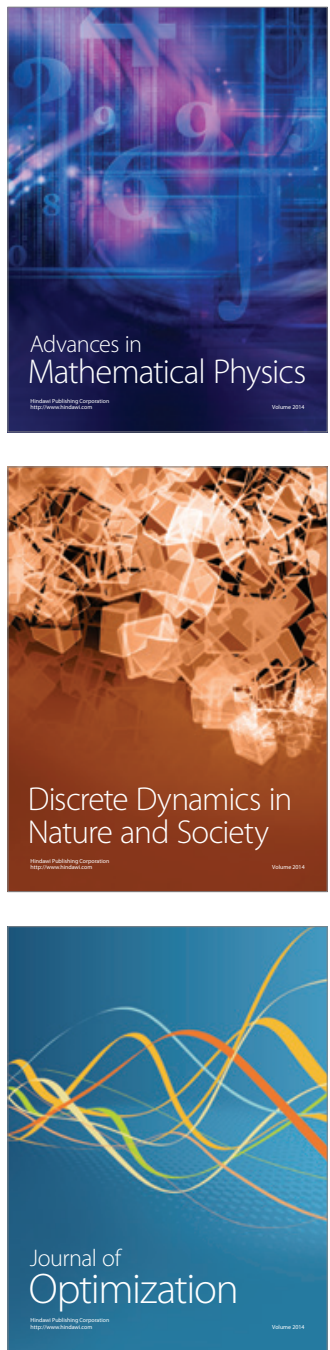